\title{
Concurrent administration of thyroxine and donepezil induces plastic changes in the prefrontal cortex of adult hypothyroid rats
}

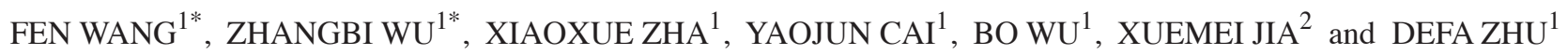 \\ ${ }^{1}$ Department of Endocrinology, Anhui Geriatric Institute, The First Affiliated Hospital of Anhui Medical University, Hefei, \\ Anhui 230022; ${ }^{2}$ Comprehensive Laboratory, College of Basic Medicine, Anhui Medical University, \\ Hefei, Anhui 230032, P.R. China
}

Received June 22, 2016; Accepted April 19, 2017

DOI: $10.3892 / \mathrm{mmr} .2017 .6977$

\begin{abstract}
The aim of the present study was to observe the effects of the concurrent administration of thyroxine (T4) and an acetylcholinesterase (AChE) inhibitor, donepezil (DON), on the hypothyroidism-induced ultrastructural changes of the prefrontal cortex (PFC) in adult rats. The acetylcholine (ACh) content and AChE activity was assessed, as well as the expressions of synaptotagmin-1 (syt-1) and SNAP-25 were analyzed in the rats. Adding $0.05 \%$ propylthiouracil to rats' drinking water induced a hypothyroid rat model. The animals were treated with T4 and DON administered separately or in combination from the fifth week. Transmission electron microscope analysis revealed that hypothyroidism induced marked ultrastructural changes, including the neurons, the synapses and the myelin sheath in the PFC. T4 or DON treatment improved the morphologic features of the PFC, and the performance of the T4 combined DON group was the closest to the control group. Moreover, hypothyroidism significantly decreased the content of ACh (29.8\%) and activity of AChE (27.8\%), which were restored to control values by $\mathrm{T} 4 \mathrm{admi}-$ nistration. In addition, DON treatment restored ACh content to normal. At the protein level, hypothyroidism increased the levels of syt-1 and SNAP-25 in the PFC, both of which were not restored to control values following T4 administration, while concurrent administration of T4 and DON was able to induce this effect. These results suggested that adult-onset hypothyroidism induce morphological, biochemical and molecular alterations in the PFC, combined administration of $\mathrm{T} 4$ and DON induce plastic changes in the PFC, different from that of the standard T4 therapy, and that the DON treatment
\end{abstract}

Correspondence to: Dr Defa Zhu, Department of Endocrinology, Anhui Geriatric Institute, The First Affiliated Hospital of Anhui Medical University, 218 Jixi Road, Hefei, Anhui 230022, P.R. China E-mail: zdfa0168@sina.com

\section{*Contributed equally}

Key words: hypothyroidism, prefrontal cortex, synaptic proteins, thyroxine, donepezil, neuroprotection may facilitate the recovery of synaptic protein impairments induced by hypothyroidism.

\section{Introduction}

The thyroid hormones (THs), including triiodothyronine (T3) and thyroxine (T4), are essential for the prefrontal cortex (PFC) structure and function (1). Insufficiency of THs in the adulthood affected performance of prefrontal learning and memory (2), in which synaptic transmission among the neurons and synaptic plasticity may be involved. Synaptic transmission is conducted by neurotransmitters released from presynaptic nerve terminals by means of $\mathrm{Ca}^{2+}$-dependent exocytosis of synaptic vesicles, and its regulation is believed to be one of the important mechanisms of synaptic plasticity underlying learning and memory (3). One neurotransmitter that may be involved is acetylcholine (ACh), whose action is dependent on its metabolizing enzyme acetylcholinesterase (AChE). Several presynaptic proteins have been implicated in presynaptic regulation of the $\mathrm{Ca}^{2+}$-dependent neurotransmission, including synaptotagmin-1 (syt-1) and synaptosomal protein of $25 \mathrm{kDa}$ (SNAP-25) (4-6). Syt-1, well established as a primary $\mathrm{Ca}^{2+}$ sensor for rapid and regulated vesicle release from neurons and neurosecretory cells $(7,8)$, directly interacts with SNAP-25 on the pre-synaptic membrane to facilitate neurotransmitter release. Thyroid dysfunction has been demonstrated to influence the cholinergic system and synaptic proteins in adult rats (9-14).

Thyroxine (T4) replacement therapy is, at present, the first choice for patients with hypothyroidism (15). However, previous clinical studies have indicated that patients with hypothyroidism who were properly treated with T4 have not always fully recovered from the symptoms affecting the central nervous system, such as the cognitive functioning and well-being (16-18). Previous animal studies of the authors have revealed that hypothyroidism induces significant expression changes of several synaptic proteins in the brain regions related to learning and memory, whereas some of the proteins were not fully restored to control values by T4 treatment $(13,14,19)$. Furthermore, in previous morphological studies, Madeira et al $(20,21)$ discovered that hypothyroidism induces reductions in the number of pyramidal cells in the hippocampal CA1 region and granule cells in the dentate 
gyrus, which were also not restored following the normalization of thyroid hormone levels in adult rats. These studies indicate that it is still necessary to discover new alternative therapeutic methods for hypothyroidism.

Donepezil (DON), as one cholinesterase inhibitor (AChEI), was primarily administered for the treatment of mild to moderate cognitive impairment. On the other hand, it was confirmed the independent neuroprotective effects in recent years $(22,23)$, which have been demonstrated in a number of models, including protection of cortical neurons in models of glutamate-induced toxicity and oxygen-glucose deprivation, and protection against the effects of hippocampal mitochondrial dysfunction in transgenic mouse models of Alzheimer's disease (AD) (24). Therefore, it is of great interest to know whether DON has neuroprotective effects on neuronal injury induced by adult hypothyroidism.

In the present study, a hypothyroid rat model was induced by adding $0.05 \%$ propylthiouracil (PTU) to their drinking water according to the authors' previous study (14). The authors observed the ultrastructure, content of ACh and the activity of AChE, as well as the expression of syt-1 and SNAP-25 in the PFC of adult hypothyroid rats, and evaluated the efficiency of T4 and DON administered separately, or in combination on the above changes in adult hypothyroid rats.

\section{Materials and methods}

Experimental animals and general protocol. The present study was conducted in strict accordance with the Animal Care and Use Committee of Anhui Medical University (Anhui, China). The protocol was approved by the Committee on the Ethics of Animal Experiments of Anhui Medical University (Anhui, China). All efforts were made to minimize suffering. Male Sprague-Dawley rats (SPF grade, ten-week-old, weighed 230-260 g) were purchased from the Experimental Animal Center of Anhui Medical University (Hefei, China). A total of $\sim 120$ rats were used for various experimental approaches in the current study. The rats were housed in groups (four to five rats per cage). All rats received a standard rodent diet and tap water ad lib. A constant temperature of $21-23^{\circ} \mathrm{C}$ and humidity of $50 \pm 5 \%$ were maintained. Following feeding for one week, the rats were randomly separated into five groups, with 24 rats in each group: i) Rats in the hypothyroid group (Hypo group) were induced by including propylthiouracil (6-n-propyl-2-thiouracil, PTU; Sigma-Aldrich; Merck KGaA, Darmstadt, Germany) in the drinking water (at a concentration of $0.05 \% \mathrm{w} / \mathrm{v}$ ) for six weeks; ii) Rats in the donepezil group (DON group) were treated with PTU for six weeks as described above, and $0.005 \%$ (w/v) donepezil (Sigma-Aldrich; Merck $\mathrm{KGaA}$ ) was added to the tap drinking water every day from the fifth week; iii) Rats in the thyroxine group (T4 group) were treated with PTU for six weeks, and were treated with daily intraperitoneal injection of T4 (dissolved in saline solution, $6 \mu \mathrm{g} / 100 \mathrm{~g}$ body weight) from the fifth week; iv) A further 20 rats in the thyroxine plus donepezil group (T4 + DON group) were treated according to the same protocols for six weeks as the T4 group beside adding $0.005 \%$ (w/v) DON to the drinking water from the fifth week; v) A total of 20 control rats in the control group (CON group) were treated with saline solution for six weeks. The total treatment time was two weeks. The dose of T4 was selected based on previous studies $(14,19)$, and the dosage was adjusted according to the rats' weights weighed weekly.

Thyroid hormones. All rats were anesthetized using chloral hydrate $(350 \mathrm{mg} / \mathrm{kg}$ body weight), following the delivery of the last dose. The blood collected from the abdominal aorta $(1.5 \mathrm{ml})$ was separated by centrifuging at $14,000 \mathrm{x} \mathrm{g}$ for 15 min (25), and then the serum was collected and rapidly frozen at $-20^{\circ} \mathrm{C}$ for subsequent analysis. Serum T3 and T4 levels were obtained using a radioimmunoassay kit (Beijing North Institute of Biological Technology, Beijing, China). All sample measurements were run in duplicate.

Sample preparation. Following the blood collection and the rats were sacrificed, the prefrontal tissues of 4 rats of each group were sampled and in $4 \%$ paraformaldehyde solution for the transmission electron microscopy (TEM) observation. The PFC of another 10 rats of each group was isolated and placed at $-80^{\circ} \mathrm{C}$, for subsequent determining the content of $\mathrm{ACh}$ and activity of $\mathrm{AChE}$. The PFC of the rest 10 rats of each group was quickly isolated and placed at $-80^{\circ} \mathrm{C}$, for western blot analysis.

TEM observation. The tissues of the PFC were cut into small pieces, $\sim 1 \mathrm{~mm}^{3}$, fixed with $2.5 \%$ glutaraldehyde at $4^{\circ} \mathrm{C}$ for 4-6 h, then fixed with $1 \%$ osmium tetroxide for $1 \mathrm{~h}$, following ethanol dehydration and epoxy resin (Epon812) embedding, the tissues were prepared the ultrathin sections, and soaked in the uranyl acetate solution and the lead citrate solution for the staining. Following rinsing, the ultrastructures were observed and photographed using a JEM-1230 TEM (Nissan Chemical Industries, Ltd., Tokyo, China).

Determination of ACh content and AChE activity. The materials and methods for determination of ACh content and AChE activity were as previously described (14). ACh levels and $\mathrm{AChE}$ activity were expressed as $\mu \mathrm{g}$ per $\mathrm{mg}$ of prefrontal protein. The protein concentration of the prefrontal homogenates was determined using a Pierce BCA Protein assay kit (Thermo Fisher Scientific, Inc., Waltham, MA, USA).

Western blot analysis. The prefrontal tissues were placed in a glass homogenizer, added radioimmunoprecipitation assay lysis buffer and phenylmethylsulfonyl fluoride, then homogenized on ice to extract the total proteins. The homogenate was then centrifuged at $15,000 \mathrm{x} \mathrm{g}$ and $4^{\circ} \mathrm{C}$ for $15 \mathrm{~min}$, the total protein concentration of the supernatant was then determined with the lorry method: Following quantification, $2 \mathrm{X}$ loading buffer (1:1) was added to the sample, proteins were denatured at $98^{\circ} \mathrm{C}$ for $10 \mathrm{~min}$, packaged and cryopreserved at $-80^{\circ} \mathrm{C}$. A total of $20 \mu \mathrm{g}$ sample of each group was subject to SDS-PAGE for $2.5 \mathrm{~h}$, then transferred onto a polyvinylidene fluoride membrane for overnight blocking with $5 \%$ skim milk. The blots were then incubated with rabbit anti-syt-1 polyclonal antibody (cat. no. ab82414; dilution, 1:1,000; Abcam, Cambridge, MA, USA), rabbit anti-SNAP-25 polyclonal antibody (cat. no. ab5666; dilution, 1:10,000; Abcam) and anti-GAPDH monoclonal antibody (cat. no. ab181602; dilution, 1:4,000; Abcam) at room temperature for $2 \mathrm{~h}$, followed by rinsing with 
A

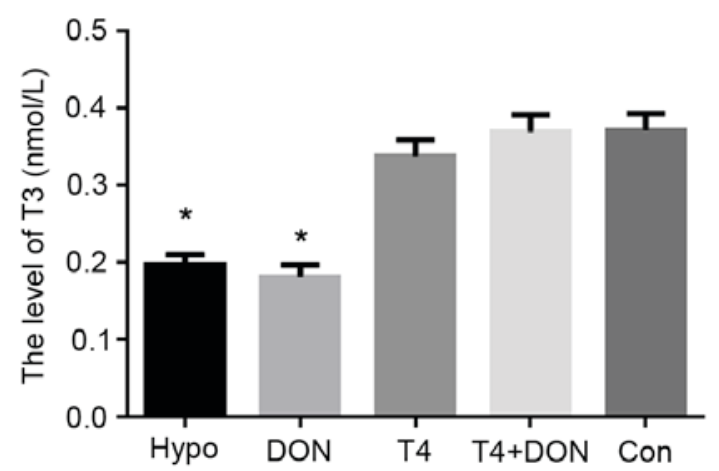

B

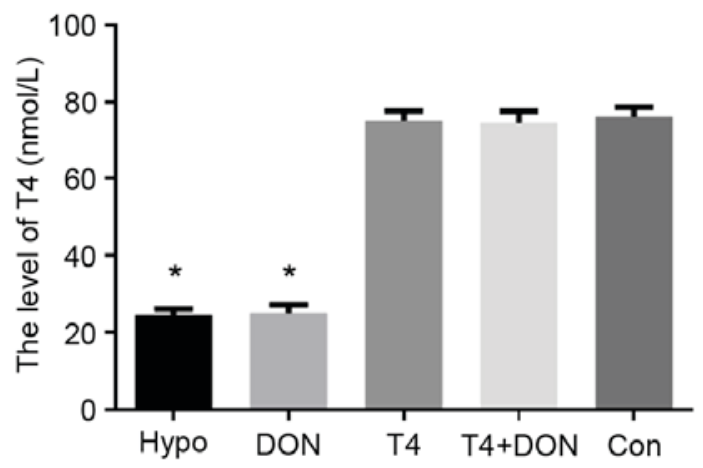

Figure 1. Serum T3 and T4 levels among the groups. The rats had significantly lower serum (A) T3 and (B) T4 levels in the Hypo and the DON group, compared to the rats in the CON group. The levels of T3 and T4 were restored to control values by T4 and T4 + DON treatment. The analysis was performed by analysis of variance. Data are presented as the mean \pm standard error of the mean. ${ }^{*} \mathrm{P}<0.05$ vs. CON group. T3, triiodothyronine; T4, thyroxine; Hypo, hypothyroid; DON, donepezil; CON, control; SEM, standard error of the mean.

$0.05 \%$ PBS-Tween 20 (PBS-T) solution three times, 10 min for each time. Membranes were then incubated with secondary antibody (cat. no. AS014; dilution, 1:200,000; horseradish peroxidase-conjugated goat-anti-rabbit IgG; ABclonal Biotech Co., Ltd., Wuhan, China) at room temperature for 1-2 h. The membrane was then rinsed with $0.05 \%$ PBS-T. Finally, the enhanced chemiluminescence solution (SuperSignal ${ }^{\mathrm{TM}}$ West Femto Maximum Sensitivity Substrate; Thermo Fisher Scientific, Inc.) was added and a Fine-do X6 visualizer was used for the photographing (Tanon Science and Technology Co., Shanghai, China). GAPDH was used as the internal reference, the ratio of optical densities of the target protein and GAPDH was then calculated using Image-Pro Plus densitometric image analysis software (version, 5.0; Media Cybernetics, Inc., Rockville, MD, USA).

Statistical analysis. Values were expressed as means \pm standard error of the mean. The data were analyzed by one-way analysis of variance using least-significant difference for post hoc analysis. $\mathrm{P}<0.05$ was used to indicate a statistically significant difference. All analyses were conducted by statistical software, SPSS (version, 13.0; SPSS, Inc., Chicago, IL, USA).

\section{Results}

Serum TH levels in rats. The serum TH levels of the five groups are presented in Fig. 1. Hypothyroidism was confirmed by measuring plasma T3 and T4 levels at the end of the experiment. Compared with the CON group, the serum T3 and T4 levels in the Hypo and the DON groups were significantly decreased $(\mathrm{P}<0.001)$. However, no significant differences in the $\mathrm{T} 3$ and T4 levels were observed in the T4 and the T4 + DON groups when compared with the CON group ( $\mathrm{P}>0.05)$.

\section{TEM observation}

Neuronal changes. In the euthyroid rats, the neurons displayed smooth and intact nuclear membranes, with chromatins evenly distributed. The mitochondria developed well, with clear internal ridge structures and the rough endoplasmic reticulum and ribosomes were rich (Fig. 2A). In the hypothyroid rats, the neurons displayed a scanty cytoplasm with few organelles, the mitochondria were characterized by highly fractured and degenerated cristae and a clear vacuolation, the endoplasmic reticulum (ER) was greatly dilated (Fig. 2B). The neurons of the DON group and the T4 group exhibited clear nuclear membranes, while the organelles were relatively sparse, and a small amount of mitochondria exhibited the vacuolation, partial rough endoplasmic reticulum was mildly dilated (Fig. 2C and D). The morphologies of neurons, mitochondria, rough ER and ribosomes of the T4 + DON group were similar to the CON group (Fig. 2E).

Synaptic changes. The structures of presynaptic membrane, synaptic cleft and postsynaptic membrane of the CON group were clear, the specialized belt was obvious, the synaptic vesicles were rich, and exhibited clear and dense-core vesicles (Fig. 3A). While the presynaptic membrane and the postsynaptic membrane of the Hypo group were fused, the synaptic vesicles were significantly reduced, and almost no clear-type synaptic vesicles could be seen (Fig. 3B). The presynaptic membrane and the postsynaptic membrane of the DON group and the T4 group were vague, the synaptic cleft was clear, while the number of clear-type synaptic vesicles was reduced (Fig. 3C and D). The structures of three synaptic layers of the $\mathrm{T} 4+\mathrm{DON}$ group were relatively clear, the synaptic vesicles were relatively rich, close to the situations of the CON group (Fig. 3E).

Myelin sheath changes. The structures of the prefrontal myelin sheath in the rats of $\mathrm{CON}$ group were complete, exhibited dense lamination, normal thickness and smooth edges (Fig. 4A). While the morphological changes of myelin sheath in the hypothroid rats showed delamination and an incompact structure, with thinner thickness and irregular outline (Fig. 4B). Following DON or T4 treatment, there was mild disruption of myelin sheath microstructures in the rats (Fig. 4C and D). The morphologies of myelin sheath of the T4 + DON group were similar to the situations of the CON group (Fig. 4E).

Content of ACh in the rat PFC. The ACh content in the PFC is illustrated in Fig. 5. The results suggested that experimentally induced hypothyroidism decreased neurotransmitter 


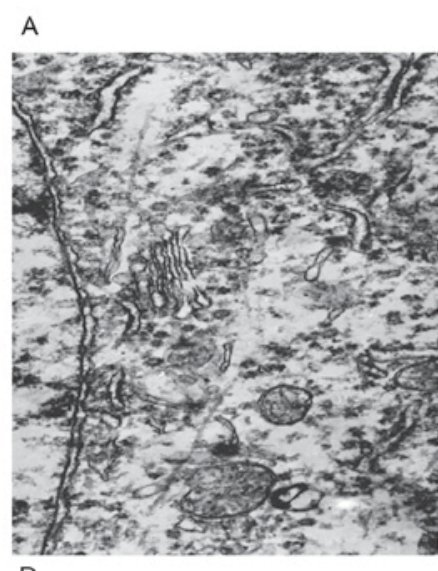

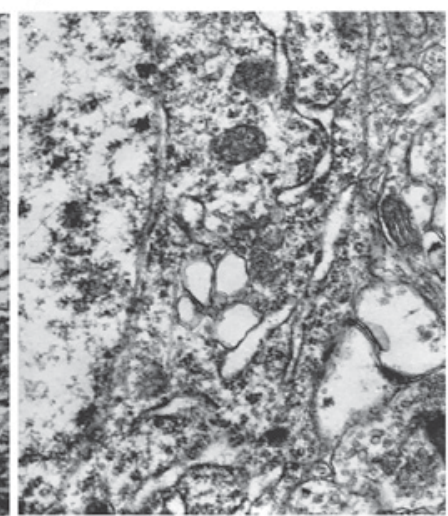

$\mathrm{E}$

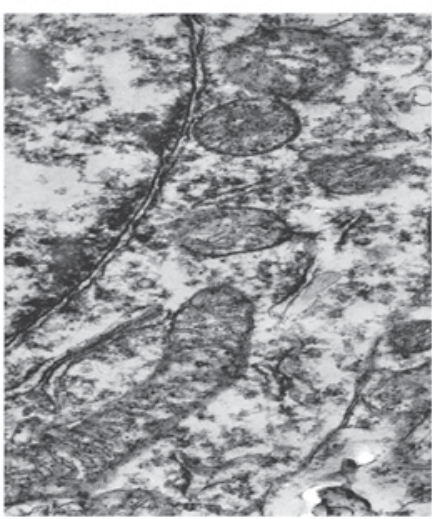

C
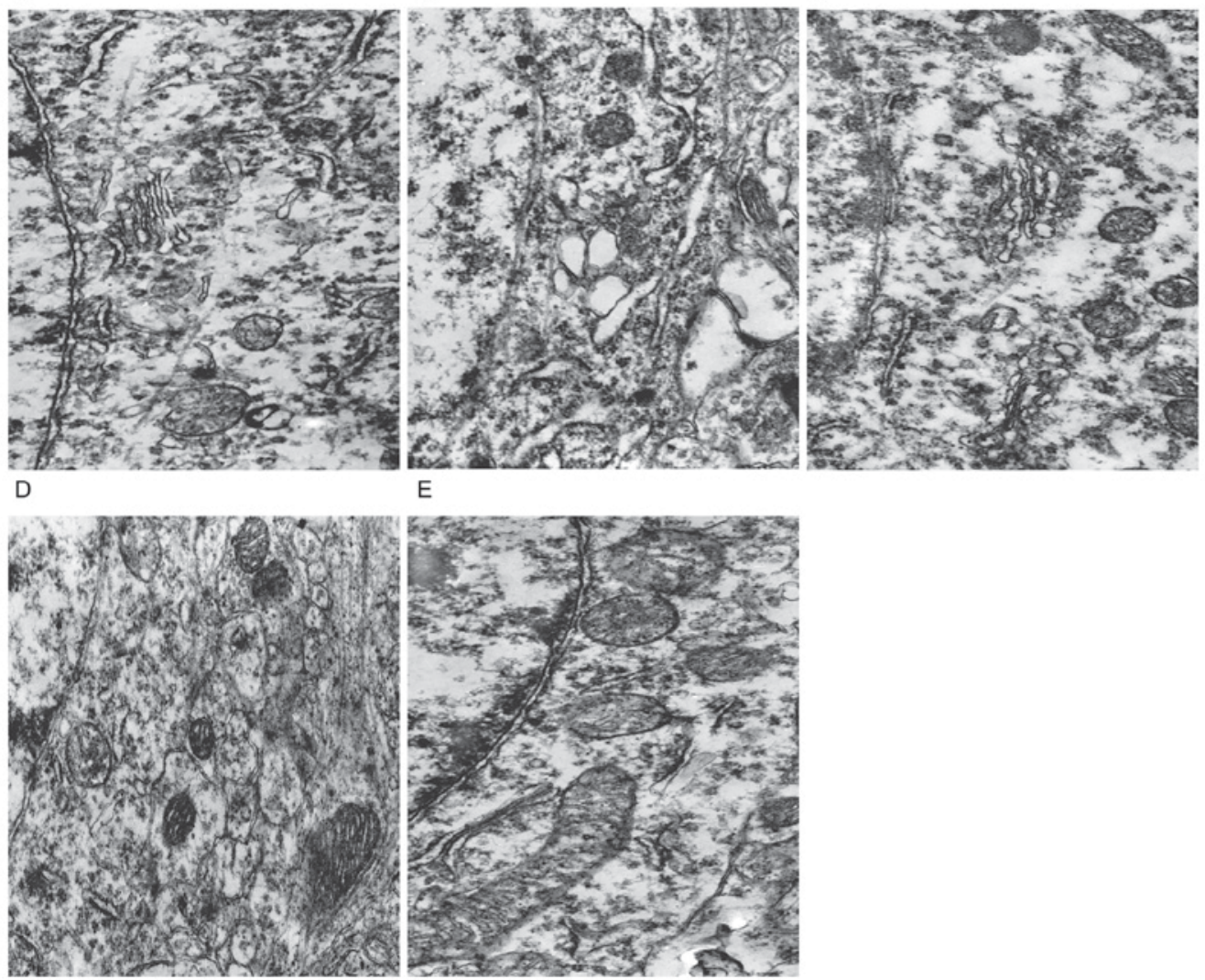

Figure 2. Representative images of the rat PFC neurons among the groups by TEM (magnification, x15,000). (A) CON group; (B) Hypo group; (C) DON group; (D) T4 group; (E) T4 + DON group. PFC, prefrontal cortex; TEM, transmission electron microscope; CON, control; Hypo, hypothyroid; DON, donepezil; T4, thyroxine.

A
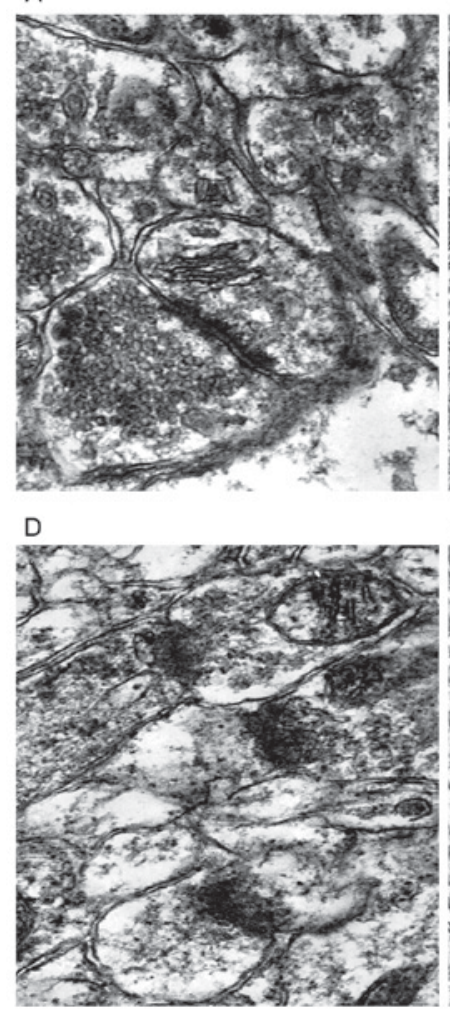

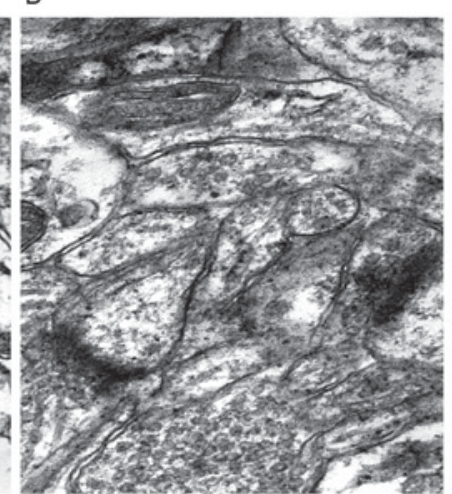

$\mathrm{E}$
C
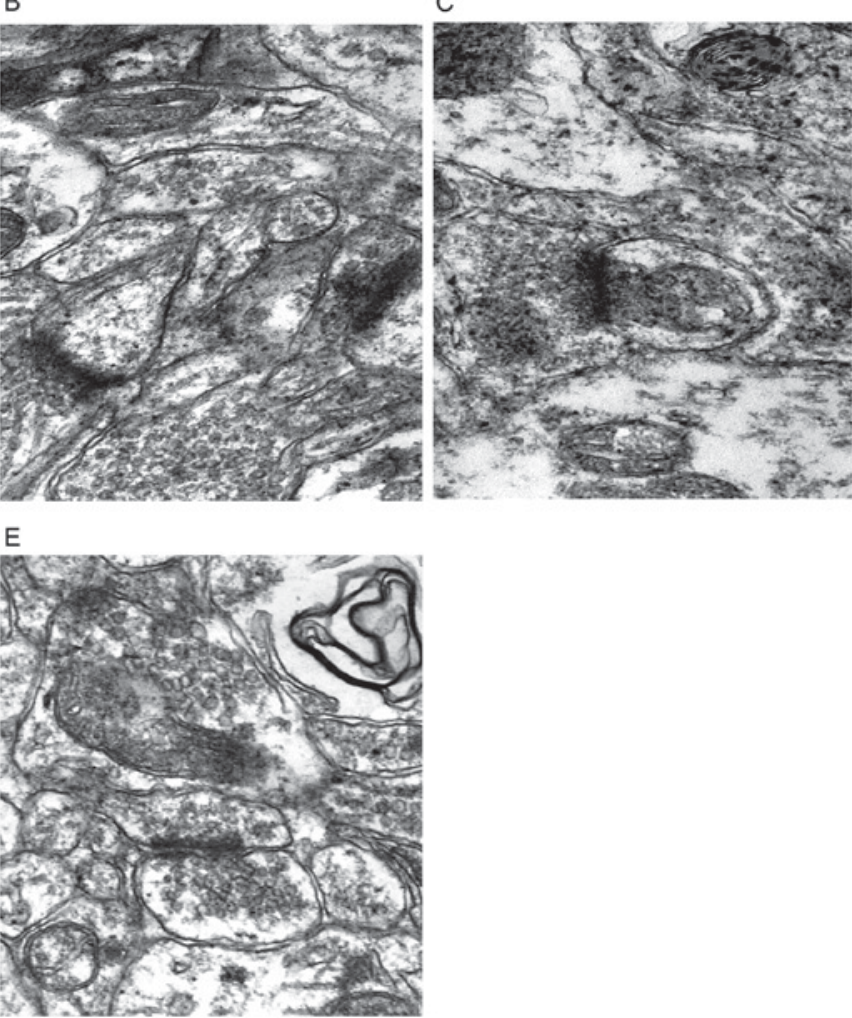

Figure 3. Representative images of the rat PFC synaptic ultrastructures among the groups by TEM (magnification, $\mathrm{x} 40,000)$. (A) CON group; (B) Hypo group; (C) DON group; (D) T4 group; (E) T4 + DON group. PFC, prefrontal cortex; TEM, transmission electron microscope; CON, control; Hypo, hypothyroid; DON, donepezil; T4, thyroxine. 


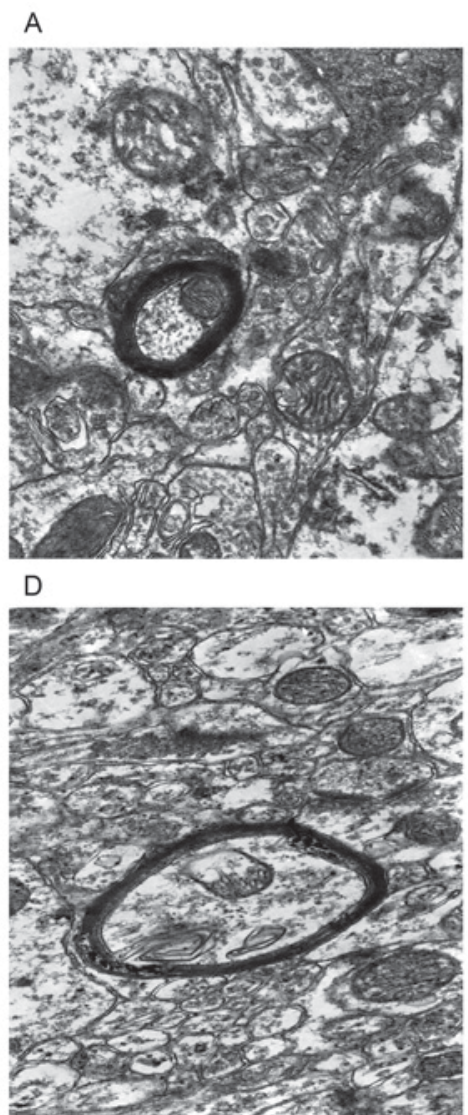

B $\quad$ C

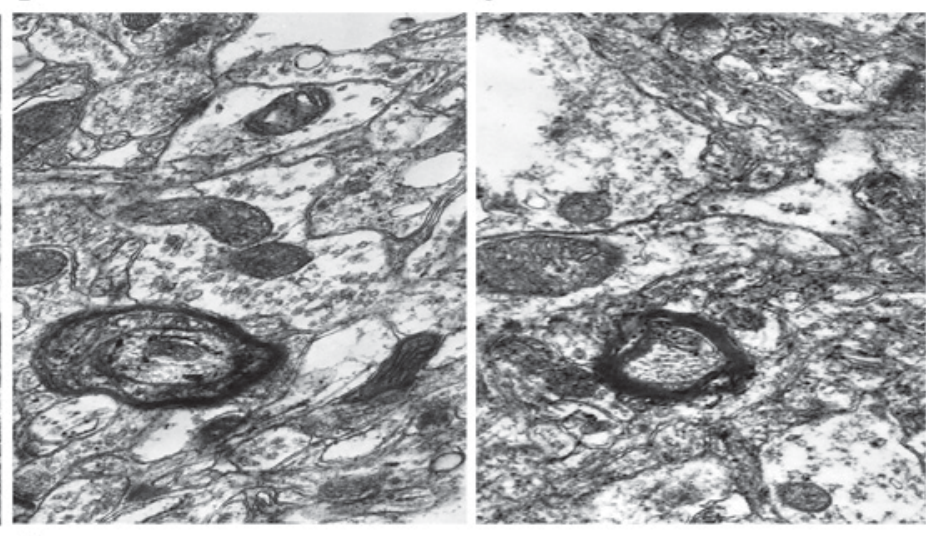

E

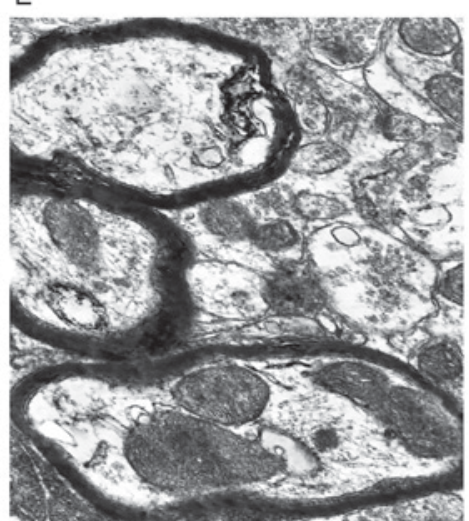

Figure 4. Representative images of the rat PFC myelin sheath among the groups by TEM (magnification, x20,000). (A) CON group; (B) Hypo group; (C) DON group; (D) T4 group; (E) T4 + DON group. PFC, prefrontal cortex; TEM, transmission electron microscope; CON, control; Hypo, hypothyroid; DON, donepezil; T4, thyroxine.

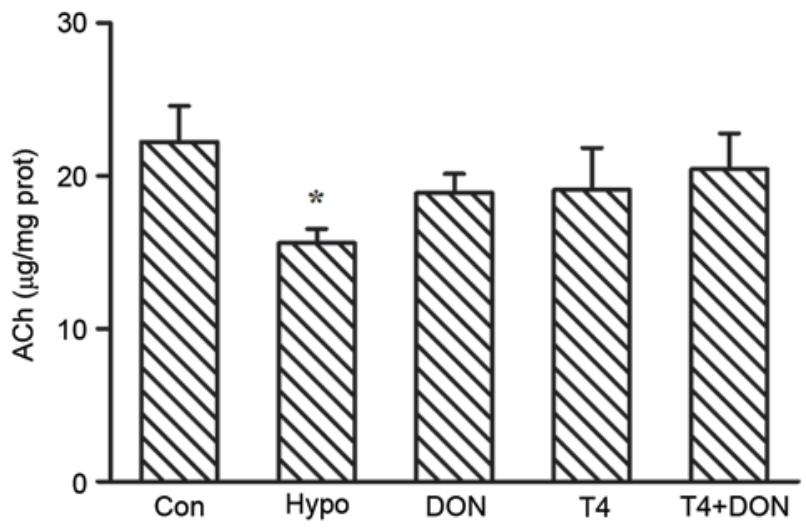

Figure 5. Concentration of prefrontal ACh from CON, Hypo, DON, T4 and T4 + DON groups $(n=10)$, homogenates were extracted from the PFC of each rat. Data are presented as means \pm standard error of the mean of three independent experiments. " $\mathrm{P}<0.05$ vs. CON. ACh, acetylcholine; T4, thyroxine; DON, donepezil; CON, control; Hypo, hypothyroid.

ACh content by $29.8 \%$ in the PFC of adult rats, as compared with the rats in the CON group $(\mathrm{P}=0.030)$. The content of ACh could be restored to control levels $(\mathrm{P}=0.237,0.291$ and 0.524 , respectively) by administration of DON, T4 or $\mathrm{T} 4+\mathrm{DON}$.

AChE activity in the rat PFC. Fig. 6 demonstrates the activity of AChE in the PFC among the groups. Compared with the

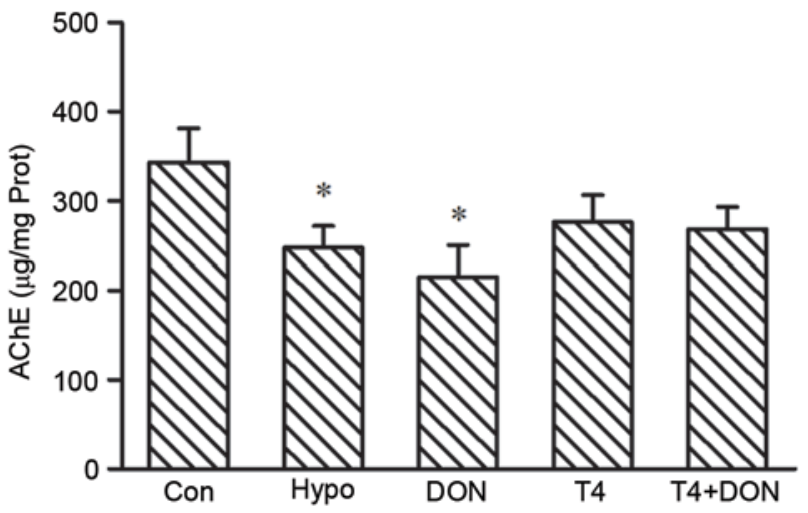

Figure 6. The AChE activity in the PFC of rats from CON, Hypo, DON, T4 and T4 + DON groups $(n=10)$, homogenates were extracted from the $\mathrm{PFC}$ of each rat. Data are presented as means \pm standard error of the mean of three independent experiments. ${ }^{*} \mathrm{P}<0.05$ vs. CON. CON, control; Hypo, hypothyroid; T4, thyroxine; DON, donepezil; AChE, acetylcholinesterase; Prot, prefrontal protein.

rats in the CON group, the AChE activity in both the Hypo and DON groups was significantly decreased by 27.8 and $37.3 \%$ $(\mathrm{P}=0.031$ and 0.010 respectively), and it was restored to control level by $\mathrm{T} 4$ treatment $(\mathrm{P}=0.144)$.

Protein levels of syt-1 and SNAP-25 in the rat PFC. Fig. 7 presents the relative levels of syt- 1 and SNAP-25 in the PFC of all rats. The results revealed that the protein expressions of 
A

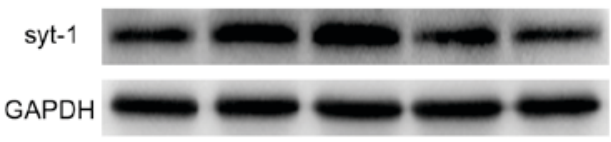

C

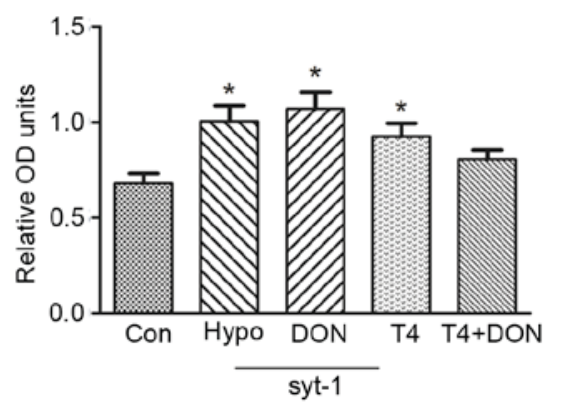

B

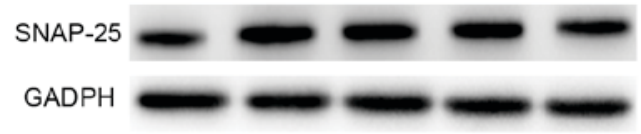

D

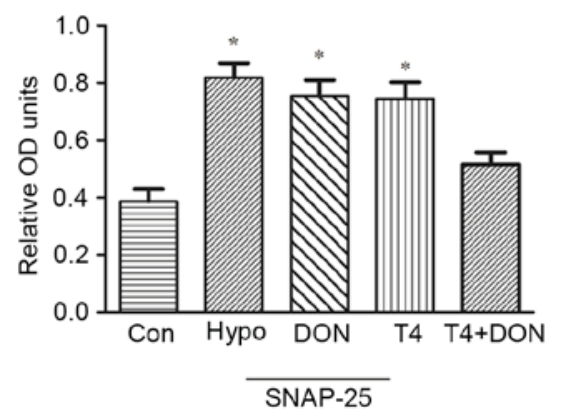

Figure 7. T4 and DON regulated the expression of syt-1 and SNAP-25 protein in the PFC of adult hypothyroid rats. (A and B) Western blot analysis of the expressions of syt-1 and SNAP-25 protein in the PFC of CON, Hypo, DON, T4 and T4 + DON groups (C and D). Quantification analysis of the relative protein levels in each group. The data are presented as the means \pm standard error of the mean $(n=10)$. " $\mathrm{P}<0.05$ vs. CON. CON, control; Hypo, hypothyroid; DON, donepezil; T4, thyroxine; syt-1, synaptotagmin-1; SNAP-25, synaptosomal-associated protein of $25 \mathrm{kDa}$; PFC, prefrontal cortex; OD, optical density.

syt-1 and SNAP-25 were severely affected by the deficiency of TH. Compared with the CON group, the prefrontal syt-1 protein expression of the Hypo Group was significantly increased by $47.8 \%(\mathrm{P}=0.002)$, and those of the DON group and the T4 group were increased by 57.4 and $36.0 \% \quad(\mathrm{P}<0.001$ and $\mathrm{P}=0.016)$, respectively, while that of the $\mathrm{T} 4+\mathrm{DON}$ group presented no difference with the $\mathrm{CON}$ group $(\mathrm{P}=0.211$; Fig. $7 \mathrm{~A}$ and C). The expression of SNAP-25 protein of the Hypo group was significantly increased than the CON group by $112 \%$ $(\mathrm{P}<0.001)$, and only increased by 95.6 and $93.3 \%$ following the DON and T4 treatment (both $\mathrm{P}<0.001$ ), respectively, following the T4 + DON treatment, the expression of SNAP-25 protein was restored to the normal level ( $\mathrm{P}=0.070$; Fig. $7 \mathrm{~B}$ and $\mathrm{D})$.

\section{Discussion}

In the present study, TEM analysis revealed that adult-onset hypothyroidism induced marked ultrastructural changes, including the neurons, the synapses and the myelin sheath in the PFC. The neurons displayed a scanty cytoplasm with few organelles, the mitochondria were characterized by highly fractured and degenerated cristae and a clear vacuolation, the endoplasmic reticulum was greatly dilated. In addition, the synapses exhibited specialized-belt fusion, the structures became unclear, and the synaptic vesicles were reduced. The morphological changes of the myelin sheath demonstrated delamination and an incompact structure, with reduced thickness and irregular outline. These morphological alterations caused by hypothyroidism have been described in other tissues, such as the hippocampus and cuneate nucleus (26-28). The morphological integrity of the neurons and the efficacy of synaptic transmission of the PFC are of primary importance for the role played by this region in various forms of learning and memory. The complete synaptic and myelin sheath morphology was the structural basis towards the information transferring among the neurons, thus the destruction of the above structures may cause the dysfunction of synaptic transmission by neurotransmitters.

It is well established that the cholinergic system is involved in higher brain functions such as learning and memory $(29,30)$. In the present study, the authors demonstrated that experimentally induced hypothyroidism decreased ACh content, as well as AChE activity, in the PFC of adult rats. A significant decrement of ACh levels has also been identified in the hippocampus and spinal cords of adult-onset hypothyroidism $(14,31)$. The AChE activity was also indicated to be decreased in the frontal cortex, the striatum, the cerebellum and entire brain of hypothyroid rats $(10,11,32,33)$. Previous research by Kundu et al (34) suggested a central T3 homeostasis that starts between the 1st and the 2nd day, and terminates between the days 18 and 20. In the present study, the ACh content and AChE activity was measured on day 42 following the adult-onset hypothyroidism in the rat PFC. Thus, the decline in ACh content and AChE activity following 42 days of PTU-induced hypothyroidism could be correlated with total disruption of the central homeostatic mechanism leading to the alteration in the neuronal functional state, primarily due to metabolic reasons. On the other hand, these changes may reflect a reduced number of cholinergic synapses in the PFC, since the PFC has been reported to be diffusely innervated by the cholinergic fibers ascending from the basal forebrain (35). However, AChE patterns still remain ambiguous as reports on both unchanged enzyme activity in thyroidectomized and increased activity in PTU-induced hypothyroid rats are available $(9,36)$.

Western blot analysis of the synaptic proteins in the PFC revealed that both syt-1 and SNAP-25 were significantly increased in the hypothyroid adult rats, compared with that of the control rats. Previous studies have demonstrated that the expression of SNAP-25 is upregulated in the dorsal and ventral hippocampus of hypothyroid mice with mild cognitive impairment (37). In addition, it was reported that in rats with 
thyroid resection, the pituitary SNAP-25 protein expression was increased (38). The increment in the expression of syt-1 was also observed in the hippocampus of neonatal hypothyroid rats (39). The regulation mechanism of the increased synaptic proteins is unknown. It is well established that these two synaptic proteins are necessary during the neurotransmitter releasing. In adult hypothyroidism, the decreased amount of the neurotransmitter ACh within the synaptic cleft may lead to an upregulation of syt-1 and SNAP-25 expression to rescue the vesicle exocytosis. However, in a previous study of the authors' (12), there is inconsistency with the information regarding the effect of adult-onset hypothyroidism on the expression of syt-1, which suggested the level of syt-1 protein was downregulated in the PFC of hypothyroid rats (three months old rats were enrolled). It is likely that, among other reasons, PTU-induced hypothyroid rats at different ages have different expression pattern of the examined synaptic protein, but the exact mechanism is still elusive.

The T4 replacement therapy was the standard solution towards the hypothyroidism treatment, which result in attenuating the alterations induced by hypothyroidism. This experiment indicated that $\mathrm{T} 4$ administration for 2 weeks resulted in amelioration of adult-onset hypothyroidism-induced morphological changes in the PFC. A previous study by Ruiz-Marcos et al (40) clearly demonstrated that adult hypothyroidism induced by surgical thyroidectomy $(\mathrm{T})$, performed at 40 or at 120 days of age, affects the pyramidal cell morphology of the visual cortex and treatment with T4 could reverse the changes. Previous studies $(41,42)$ also indicated that treatment of the $\mathrm{T}$ rats performed at 10 days of age with T4 starting at 12 days of age ( 2 days following $\mathrm{T}$ ) prevented the morphological alterations in the visual cortex, as assessed in 40-day-old animals. In 60-day-old animals, the recovery was not complete, though treatment with T4 starting at 12 or 15 days of age had an ameliorating effect on the pyramids. However, in another work of the pyramidal cortical cell morphology studied by multivariate analysis, treatment with T4 started at 12 days of age did not prevent the changes due to $\mathrm{T}$ performed at 10 days of age (43). In the present work, the authors observed a clear-cut ameliorating effect of T4 administration on adult-onset hypothyroidism-induced morphological changes in the PFC. Detailed quantitative data on the effects of adult hypothyroidism and T4 substitution therapy upon the prefrontal morphological parameters is of great interest for the future studies. In addition, treatment of the adult hypothyroid rats with $\mathrm{T} 4$ was revealed to replenish the decreased ACh levels and AChE activity in the PFC to control values. The reversible changes of the ACh concentration and $\mathrm{AChE}$ activity by $\mathrm{T} 4$ were also observed in other brain regions, such as the hippocampus of the adult hypothyroid rats (14). The results above indicated that $\mathrm{ACh}$ and $\mathrm{AChE}$ may be under direct thyroid hormone control. Indeed, previous studies from tissue culture experiments revealed that as T3 levels approach the level of total TH in the blood, the activities of acetylcholine transferase, the enzyme responsible for the synthesis of $\mathrm{ACh}$, and AChE were markedly enhanced $(44,45)$.

Regretfully, the present results demonstrated that T4 monotherapy for 2 weeks does not restore the expression levels of syt-1 and SNAP-25, which is consistent with a previous work by the author (14). However, the exact mechanism underlying this remained elusive. It may be that either the changes in the synaptic proteins are irreversible or that the restoration could be achieved only by much longer treatment or much larger dose of T4 treatment. However, a previous study indicated that even T4 treatment for six weeks failed to return the decreased PKC $\gamma$ level back to normal (46). In a previous study, the authors demonstrated that, when given large-dose T4 shock therapy to the adult hypothyroid rats, the hippocampal syt-1 and SNAP-25 proteins could restore to the normal levels, but hyperthyroidism could be induced at the same time (13). Alternatively, despite achieving a biochemically euthyroid state, some patients with hypothyroidism continue to complain of significant fatigue, weight issues, and diminished neurocognitive function, perceived to be due to inadequately treated hypothyroidism (16). Thus, a therapy combining the administration of T4 and T3 was recommended by some practitioners and investigators for patients with hypothyroidism. However, multiple studies have identified no significant differences in various quality-of-life measures (for example: Depression, fatigue, cognitive function) with use of the combination of T4 and T3 compared to T4 alone (15). The findings above indicated that it is still necessary to discover new alternative therapeutic methods for treatment of hypothyroidism. DON, as a AChEI, has demonstrated efficacy in improving cognitive function and providing neuroprotective effects (23).

Previously, the authors first explored the efficiency of T4 and DON on hippocampal ACh content, AChE activity as well as expression changes of several synaptic proteins, such as syt-1, SNAP-25, munc-18 and syntaxin-1 induced by adult-onset hypothyroidism, the authors' results demonstrated that $\mathrm{T} 4$ and $\mathrm{DON}$ administered in combination resulted in more effective restoration levels of $\mathrm{ACh}$ and synaptic proteins than either alone $(14,19)$. In the present work, the authors observed that, following the T4 + DON treatment, the morphological alterations, including the neurons, the synapses and the myelin sheath in the PFC were similar to that of the CON group. In addition, the expression levels of ACh, syt-1and SNAP-25 were closer to the levels of the control group, which is consistent with previous findings. These results suggested that DON treatment resulted in amelioration of impairments induced by hypothyroidism, although the exact mechanisms underlying the regulation remain largely uncharacterized. DON, as an AChEI, has been previously used to compensate for ACh depletion in the AD brain. In the current study, the decreased levels of ACh induced by hypothyroidism were restored by DON treatment for 2 weeks. Similar results were observed in studies revealing that other AChEIs, including galantamine and physostigmine increase ACh levels $(47,48)$. Therefore, it is probable that DON increases ACh levels through the well-established mechanism of preventing the enzymatic degradation of $\mathrm{ACh}$, thus prolonging its availability. Some clinical and experimental studies, however, have suggested that DON also provide neuroprotection. Of note, following the single DON treatment, the morphological alterations, including the neurons, the synapses and the myelin sheath in the PFC of the hypothyroid rats recovered to some extent. Previous studies have reported that the neuroprotective effects of DON prevent the alterations of the neuronal dendrite morphology in the PFC caused by aging (49), protect neurons 
from ischemic damage, glutamate-induced neurotoxicity and amyloid $\beta$ neurotoxicity (50). Furthermore, it has been demonstrated that DON treatment can preserve presynaptic protein expression in the hippocampus and spinal cord in a tauopathy mouse model (23). As indicated in previous studies, neuroprotective effects of DON may be mediated by many mechanisms, including an increased expression of AChRs, activation of the nAChR/PI3K pathway (51-53) and the o1 receptor/PLC/PKC pathway (54).

Taken together, the present findings demonstrate that hypothyroidism could cause pathological damages in the prefrontal ultrastructures, induce alterations of prefrontal ACh level and AChE activity, as well as the expression changes of syt-1 and SNAP-25 level, in adult rats. Combined administration of T4 and DON induce plastic changes in the PFC, different from that of T4 monotherapy, and suggest that the DON treatment may facilitate the recovery of the synaptic protein impairments induced by hypothyroidism.

\section{Acknowledgments}

The authors would like to thank the Department of Toxicology of Anhui Medical University (Anhui, China) was greatly appreciated for the technical assistance. The present study was financially supported by the National Natural Science Foundation of China (grant no. 81272152).

\section{References}

1. Dugbartey AT: Neurocognitive aspects of hypothyroidism. Arch Intern Med 158: 1413-1418, 1998.

2. Zhu DF, Wang ZX, Zhang DR, Pan ZL, He S, Hu XP, Chen XC and Zhou JN: fMRI revealed neural substrate for reversible working memory dysfunction in subclinical hypothyroidism. Brain 129: 2923-2930, 2006.

3. Jessell TM and Kandel ER: Synaptic transmission: A bidirectional and self-modifiable form of cell-cell communication. Cell 72 (Suppl): S1-S30, 1993.

4. Zhou Q, Lai Y, Bacaj T, Zhao M, Lyubimov AY, Uervirojnangkoorn M, Zeldin OB, Brewster AS, Sauter NK, Cohen AE, et al: Architecture of the synaptotagmin-SNARE machinery for neuronal exocytosis. Nature 525: 62-67, 2015.

5. Mehta PP, Battenberg E and Wilson MC: SNAP-25 and synaptotagmin involvement in the final $\mathrm{Ca}^{(2+)}$-dependent triggering of neurotransmitter exocytosis. Proc Natl Acad Sci USA 93: 10471-10476, 1996.

6. de Wit H, Walter AM, Milosevic I, Gulyás-Kovács A, Riedel D, Sørensen JB and Verhage M: Synaptotagmin-1 docks secretory vesicles to syntaxin-1/SNAP-25 acceptor complexes. Cell 138: 935-946, 2009

7. Sudhof TC: The synaptic vesicle cycle. Annu Rev Neurosci 27: 509-547, 2004

8. Chapman ER: How does synaptotagmin trigger neurotransmitter release? Annu Rev Biochem 77: 615-641, 2008.

9. Salvati S, Attorri L, Campeggi LM, Olivieri A, Sorcini M, Fortuna S and Pintor A: Effect of propylthiouracil-induced hypothyroidism on cerebral cortex of young and aged rats: Lipid composition of synaptosomes, muscarinic receptor sites, and acetylcholinesterase activity. Neurochem Res 19: 1181-1186, 1994.

10. Carageorgiou H, Pantos C, Zarros A, Mourouzis I, Varonos D, Cokkinos D and Tsakiris S: Changes in antioxidant status, protein concentration, acetylcholinesterase, $\left(\mathrm{Na}^{+}, \mathrm{K}^{+}\right)-$, and $\mathrm{Mg} 2^{+}-$ATPase activities in the brain of hyper- and hypothyroid adult rats. Metab Brain Dis 20: 129-139, 2005.

11. Carageorgiou H, Pantos C, Zarros A, Stolakis V, Mourouzis I, Cokkinos D and Tsakiris S: Changes in acetylcholinesterase, $\mathrm{Na}^{+}, \mathrm{K}^{+}$-ATPase, and $\mathrm{Mg}^{2+}$-ATPase activities in the frontal cortex and the hippocampus of hyper- and hypothyroid adult rats. Metabolism 56: 1104-1110, 2007.
12. Yang HY, Sun CP, Jia XM, Gui L, Zhu DF and Ma WQ: Effect of thyroxine on SNARE complex and synaptotagmin-1 expression in the prefrontal cortex of rats with adult-onset hypothyroidism. J Endocrinol Invest 35: 312-316, 2012.

13. Liu CL, Xu YX, Zhan Y, Hu HL, Jia XM, Chen GH and Zhu DF: Effect of thyroxine on synaptotagmin 1 and SNAP-25 expression in dorsal hippocampus of adult-onset hypothyroid rats. J Endocrinol Invest 34: 280-286, 2011.

14. Wang F, Zeng X, Zhu Y, Ning D, Liu J, Liu C, Jia X and Zhu D: Effects of thyroxine and donepezil on hippocampal acetylcholine content, acetylcholinesterase activity, synaptotagmin-1 and SNAP-25 expression in hypothyroid adult rats. Mol Med Rep 11: 775-782, 2015.

15. No authors listed: Drugs for hypothyroidism. Med Lett Drugs Ther 57: 147-150, 2015.

16. Wekking EM, Appelhof BC, Fliers E, Schene AH, Huyser J, Tijssen JG and Wiersinga WM: Cognitive functioning and well-being in euthyroid patients on thyroxine replacement therapy for primary hypothyroidism. Eur J Endocrinol 153: 747-753, 2005.

17. Samuels MH, Schuff KG, Carlson NE, Carello $P$ and Janowsky JS: Health status, psychological symptoms, mood, and cognition in L-thyroxine-treated hypothyroid subjects. Thyroid 17: 249-258, 2007.

18. Saravanan P, Chau WF, Roberts N, Vedhara K, Greenwood R and Dayan CM: Psychological well-being in patients on 'adequate' doses of 1-thyroxine: Results of a large, controlled community-based questionnaire study. Clin Endocrinol (Oxf) 57: 577-585, 2002 .

19. Wang N, Cai Y, Wang F, Zeng X, Jia X, Tao F and Zhu D: Effects of thyroxin and donepezil on hippocampal acetylcholine content and syntaxin- 1 and munc-18 expression in adult rats with hypothyroidism. Exp Ther Med 7: 529-536, 2014.

20. Madeira MD, Sousa N, Lima-Andrade MT, Calheiros F, Cadete-Leite A and Paula-Barbosa MM: Selective vulnerability of the hippocampal pyramidal neurons to hypothyroidism in male and female rats. J Comp Neurol 322: 501-518, 1992.

21. Madeira MD, Cadete-Leite A, Andrade JP and Paula-Barbosa MM: Effects of hypothyroidism upon the granular layer of the dentate gyrus in male and female adult rats: A morphometric study. J Comp Neurol 314: 171-186, 1991.

22. Pepeu G and Giovannini MG: Cholinesterase inhibitors and beyond. Curr Alzheimer Res 6: 86-96, 2009.

23. Yoshiyama Y,Kojima A,Ishikawa C and Arai K: Anti-inflammatory action of donepezil ameliorates tau pathology, synaptic loss and neurodegeneration in a tauopathy mouse model. J Alzheimers Dis 22: 295-306, 2010.

24. Riepe MW: Cholinergic treatment: What are the early neuropathological targets? Eur J Neurol 12 (Suppl 3): S3-S9, 2005.

25. Gerges NZ, Stringer JL and Alkadhi KA: Combination of hypothyroidism and stress abolishes early LTP in the CA1 but not dentate gyrus of hippocampus of adult rats. Brain Res 922: 250-260, 2001.

26. Cortes C, Eugenin E, Aliaga E, Carreño LJ, Bueno SM, Gonzalez PA, Gayol S, Naranjo D, Noches V, Marassi MP, et al: Hypothyroidism in the adult rat causes incremental changes in brain-derived neurotrophic factor, neuronal and astrocyte apoptosis, gliosis, and deterioration of postsynaptic density. Thyroid 22: 951-963, 2012.

27. Madeira MD and Paula-Barbosa MM: Reorganization of mossy fiber synapses in male and female hypothyroid rats: A stereological study. J Comp Neurol 337: 334-352, 1993.

28. David S and Nathaniel EJ: Neuronal changes induced by neonatal hypothyroidism: An ultrastructural study. Am J Anat 167: 381-394, 1983.

29. Gold PE: Acetylcholine modulation of neural systems involved in learning and memory. Neurobiol Learn Mem 80: 194-210, 2003.

30. Bartus RT, Dean RL III, Beer B and Lippa AS: The cholinergic hypothesis of geriatric memory dysfunction. Science 217 : 408-414, 1982.

31. Molinengo L, Cassone MC and Oggero L: Action of hypo- and hyperthyroidism on the postmortal decay of acetylcholine in the rat spinal cord. Neuroendocrinology 42: 28-31, 1986.

32. Virgili M, Saverino O, Vaccari M, Barnabei O and Contestabile A: Temporal, regional and cellular selectivity of neonatal alteration of the thyroid state on neurochemical maturation in the rat. Exp Brain Res 83: 555-561, 1991.

33. Carageorgiou H, Pantos C, Zarros A, Stolakis V, Mourouzis I, Cokkinos D and Tsakiris S: Effects of hyper- and hypothyroidism on acetylcholinesterase, $\left(\mathrm{Na}\left(^{+}\right), \mathrm{K}\left({ }^{+}\right)\right)$- and $\mathrm{Mg}\left({ }^{2+}\right)$-ATPase activities of adult rat hypothalamus and cerebellum. Metab Brain Dis 22: 31-38, 2007. 
34. Kundu S, Pramanik M, Roy S, De J, Biswas A and Ray AK Maintenance of brain thyroid hormone level during peripheral hypothyroid condition in adult rat. Life Sci 79: 1450-1455, 2006.

35. Semba K: Phylogenetic and ontogenetic aspects of the basal forebrain cholinergic neurons and their innervation of the cerebral cortex. Prog Brain Res 145: 3-43, 2004.

36. Owasoyo JO, Egbunike GN and Iramain CA: The influence of thyroid gland and thyroxine on the acetylcholinesterase activity of rat brain and adenohypophysis. Endokrinologie 77: 242-246, 1981

37. Cao L, Jiang W, Wang F, Yang QG, Wang C, Chen YP and Chen GH: The reduced serum free triiodothyronine and increased dorsal hippocampal SNAP-25 and Munc18-1 had existed in middle-aged CD-1 mice with mild spatial cognitive impairment. Brain Res 1540: 9-20, 2013

38. Quintanar JL and Salinas E: Effect of hypothyroidism on synaptosomal-associated protein of $25 \mathrm{kDa}$ and syntaxin-1 expression in adenohypophyses of rat. J Endocrinol Invest 25: 754-758, 2002.

39. Vara H, Martínez B, Santos A and Colino A: Thyroid hormone regulates neurotransmitter release in neonatal rat hippocampus. Neuroscience 110: 19-28, 2002

40. Ruiz-Marcos A, Sánchez-Toscano F, Escobar del Rey F and Morreale de Escobar G: Reversible morphological alterations of cortical neurons in juvenile and adult hypothyroidism in the rat. Brain Res 185: 91-102, 1980.

41. Ruiz-Marcos A, Salas J, Sanchez-Toscano F, Escobar del Rey F and Morreale de Escobar G: Effect of neonatal and adult-onset hypothyroidism on pyramidal cells of the rat auditory cortex. Brain Res 285: 205-213, 1983.

42. Ruiz-Marcos A, Sanchez-Toscano F, Escobar del Rey F and Morreale de Escobar G: Severe hypothyroidism and the maturation of the rat cerebral cortex. Brain Res 162: 315-329, 1979.

43. Ipina SL, Ruiz-Marcos A, Escobar del Rey F and Morreale de Escobar G: Pyramidal cortical cell morphology studied by multivariate analysis: Effects of neonatal thyroidectomy, ageing and thyroxine-substitution therapy. Brain Res 465: 219-229, 1987.

44. Honegger P and Lenoir D: Triodothyronine enhancement of neuronal differentiation in aggregating fetal rat brain cells cultured in a chemically defined medium. Brain Res 199: 425-434, 1980.
45. Akuzawa K and Wakabayashi K: A serum-free culture of the neurons in the septal, preoptic and hypothalamic region. Endocrinol Jpn 32: 163-173, 1985.

46. Alzoubi KH, Gerges NZ and Alkadhi KA: Levothyroxin restores hypothyroidism-induced impairment of LTP of hippocampal CA1: Electrophysiological and molecular studies. Exp Neurol 195: 330-341, 2005.

47. Saiyed M and Riker WK: Cholinergic and anticholinergic drug effects on survival during hypoxia: Significant gender differences. J Pharmacol Exp Ther 264: 1146-1153, 1993.

48. Dimitrova DS and Getova-Spassova DP: Effects of galantamine and donepezil on active and passive avoidance tests in rats with induced hypoxia. J Pharmacol Sci 101: 199-204, 2006.

49. Alcantara-Gonzalez F, Juarez I, Solis O, Martinez-Tellez I, Camacho-Abrego I, Masliah E, Mena R and Flores G: Enhanced dendritic spine number of neurons of the prefrontal cortex, hippocampus and nucleus accumbens in old rats after chronic donepezil administration. Synapse 64: 786-793, 2010.

50. Akasofu S, Kimura M, Kosasa T, Sawada K and Ogura H: Study of neuroprotection of donepezil, a therapy for Alzheimer's disease. Chem Biol Interact 175: 222-226, 2008.

51. Akaike A, Takada-Takatori $\mathrm{Y}$, Kume $\mathrm{T}$ and Izumi $\mathrm{Y}$ Mechanisms of neuroprotective effects of nicotine and acetylcholinesterase inhibitors: Role of alpha4 and alpha7 receptors in neuroprotection. J Mol Neurosci 40: 211-216, 2010.

52. Shen H, Kihara T, Hongo H, Wu X, Kem WR, Shimohama S, Akaike A, Niidome T and Sugimoto H: Neuroprotection by donepezil against glutamate excitotoxicity involves stimulation of alpha7 nicotinic receptors and internalization of NMDA receptors. Br J Pharmacol 161: 127-139, 2010.

53. Takada-Takatori Y, Kume T, Izumi Y, Ohgi Y, Niidome T, Fujii T, Sugimoto H and Akaike A: Roles of nicotinic receptors in acetylcholinesterase inhibitor-induced neuroprotection and nicotinic receptor up-regulation. Biol Pharm Bull 32: 318-324, 2009

54. Meunier J, Ieni J and Maurice T: The anti-amnesic and neuroprotective effects of donepezil against amyloid beta25-35 peptide-induced toxicity in mice involve an interaction with the sigma1 receptor. Br J Pharmacol 149: 998-1012, 2006. 